\title{
A comparative study of PEEC circuit elements computation
}

\author{
Giulio Antonini*, J. Ekman† \\ Antonio Ciccomancini Scogna*, Albert E. Ruehli§ \\ * University of L'Aquila, Poggio di Roio, 67040 AQ, Italy \\ $\dagger$ EISLAB, Luleå University of Technology, SE-971 87 Luleå, Sweden \\ § IBM Research Division, Yorktown Heights, NY 10598, USA
}

\begin{abstract}
A key use of the PEEC method is the solution of combined electromagnetic and circuit problems as they occur in many situations in todays very large scale integrated circuits (VLSI) and systems. An important aspect of this approach is the fast and accurate computation of PEEC circuit matrix elements, the partial inductances and normalized coefficients of potential. Recently, Fast Multipole Methods (FMM) have been applied to the PEEC method in the frequency domain as a way to speed up the solution. In this paper, we consider the fast evaluation of the PEEC circuit matrix elements by two different methods, a matrix version of the (FMM)PEEC method and a method which we call the Fast Multi-Function (FMF)PEEC approach. In this technique, the matrix coefficients are evaluated using analytical functions approximation of the coefficients in combination with a proper choice of numerical quadrature formulas.
\end{abstract}

\section{Introduction}

Today, a multitude of mixed electromagnetic and circuit problems must be solved which are of an ever increasing size. Also, the problems which can be solved with quasistatic approaches are steadily decreasing as the frequencies increase and the rise times decrease. Simultaneously, compute times are becoming too large especially in the frequency domain where the entire problem is coupled at low frequencies, independent of the problem size. Further, twenty subdivisions are required in each spatial direction, at the shortest wavelength in the spectrum, for accurate impedance results. This leads to very large matrices especially for electrically large problem sizes. The Partial Element Equivalent Circuit (PEEC) method has become a very popular approach for the solution of the mixed EM and circuits problems due to its flexibility and since can use the same general Modified Nodal Analysis (MNA) solution techniques which are used in most Spice type circuit solvers. In this paper, we consider the fast evaluation of the PEEC circuit matrix elements by two different methods, a matrix version of the (FMM)PEEC method and a method which we call the Fast MultiFunction (FMF)PEEC approach. In this technique, the matrix coefficients are evaluated using analytical functions approximation of the coefficients in combination with a proper choice of numerical quadrature formulas.

We show that with a straight forward evaluation of the matrix elements, which are the PEEC circuit parameters, the partial inductances and normalized potential coefficients evaluation can be very time consuming. Further, for the solution of dense problems with the PEEC method, we require very high accuracy for these circuit elements since it strongly impacts the accuracy of the results. Two factors are of key importance for the compute time. Using a straight forward numerical approach for the evaluation of each partial inductance for the general case can be very time consuming due to the six fold integral which needs to be evaluated. If, in a numerical evaluation, we subdivide each direction into $M$ sections, then this is an $O\left(M^{6}\right)$ process. It is clear that this evaluation must be done with upmost care to conserve compute time. This is where part of the time saving results from in the FMF approach. The second issue is the evaluation of close to $O\left(N^{2}\right)$ elements for a mostly dense circuit matrix where $N$ is the matrix size. Of course, this is the case where the problem involves predominantly PEEC elements and only a few conventional lumped circuit elements. The Fast Multipole Method FMM has been developed to reduce the $O\left(N^{2}\right)$ evaluations. Here, we look at both approaches to come up with a comparison of the approaches. In all these methods, we want to keep both the matrix evaluation and matrix solution time small. However, in this short paper we are concerned only with fast matrix element evaluation techniques and not the solution of the matrix system at hand.

The fast element evaluation techniques considered in this paper address the frequency domain only, but the approaches, with some modifications, apply to the time domain as well. Aspects of FMF have been practiced by a multitude of researchers in different forms starting with the so called subarea method where all off diagonal matrix 
elements used collocation point approximations. Today, we know that the accuracy of this method is inadequate for PEEC. As is always the case, the FMF and the FMM approaches perform best for different problems. Hierarchy can play a considerable role in the speed up of both fundamental methods, provided that the geometry is suitable for a geometrical decomposition. However, the best range of applications have not been clearly identified since the methods are usually applied to a specific class of problem. Hence, much more work is required to classify the approaches. Here, we consider briefly the FMF methods and then in somewhat more detail the FMM type methods.

\section{Fast multi-function evaluation of coefficients}

It is important to recognize that for maximum efficiency, a mixture of different approaches is required. Importantly, the solution method need to be tailored to the type of problem at hand to minimize compute time. FMF methods have been used in PEEC from the start to reduce the solution time. The circuit element accuracy requirements must be met with the least compute time. For example, for special purpose EM solvers for non dense problems less accuracy is required. In general, many researchers have been in part using such approaches. A multitude of analytical as well as numerical solution methods are used for general purpose PEEC solvers. For example, long, thin conductors are simple to approximate analytically and are difficult to model with conventional multipoles. Hence, different formulas are used which have to take the shape of the conductors into account in the calculation of partial inductances and potential coefficients. A key problem with a general purpose PEEC solver is the very high accuracy required for the near coefficient for dense problems. Five to six digits of accuracy is needed for the self term while the evaluation of far coefficients can be less accurate without loss of overall solution accuracy. In the limited space available in this paper, we outline a few steps of a control mechanism for a variable order Gauss-Legendre quadrature for speeding up the far coefficient evaluation. This is only part of the overall FMF approach.

We assume that the space discretization is carried out such that the size of the largest inductive and capacitive cell is less than $\lambda_{\min } / 20$ where $\lambda_{\min }$ is the minimum wavelength corresponding to the maximum frequency in the excitation spectrum. The algorithm which is also applied to the non-orthogonal case is based on the distance and shape of the two cells involved. Compute the following quantities:

1. find the maximum size, maxSize, of the cells;

2 . find the center to center distance, $R_{c 2 c}$, between the two cells;
3. find the ratio, farRatio $=R_{c 2 c} / \max$ Size;

4. if (farRatio $>30$ ) center to center point approximation is used

else a more accurate representation is needed. An example is given below.

If farRatio $\leq 30$ a Gauss-Legendre quadrature can be used with a properly chosen order. For this, the gaps EdgeDist between the edges of cells are evaluated in each direction $i=x, y, z$. Finally a 3 -dimensional parameter is evaluated for each direction $i$ as distRat di $_{i}=E d g e D i s t_{i} /$ Size $_{i}$ for $i=x, y, z$. The following decision algorithm is applied to choose the order:
1. if $\left(\right.$ distRat $\left._{i}<1\right)$
$O r d e r_{i}=6$
2. if $\left(\right.$ dist Rat $\left._{i}<3\right)$
$O$ rder $_{i}=5$
3. if (distRat d $_{i}<10$ )
Order $_{i}=4$
4. if (distRat d $\left._{i}<20\right)$
Order $_{i}=3$
5. else
Order $_{i}=2$

for $i=x, y, z$, for each cell It is clear that the geometrical hierarch can be utilized for the FMF to speed up matrix element calculation. Sections of a geometry can be utilized to speed up the element evaluation and the geometrical date can also be deduced directly from the hierarchy. Then, we do get similar properties in the storage requirements and evaluation time in some cases as the FMM approach. This emphasizes the requirement for a clear understanding of the utility of the techniques.

\section{Fast multipole approach for par- tial elements}

The fast multipole method FMM, based computation of PEEC circuit elements, can also result in a significant CPU-time saving. The FMM has been widely used to accelerate the solution of scattering problems $[1,2]$. The key compute time reduction result from the breakup of the coefficients into three different parts, all of which do not directly depend on both the source and observation points. This circumvents the computation of $N^{2}$ interactions. The cost of computing each function is usually more expensive than in the equivalent FMF. However, each of the computed functions are used multiple times. The overall efficiency of the FMM depends on different aspects of the problem at hand than is the case for the FMF. This type of behavior makes the methods complementary in many ways.

The basic idea of FMM is to subdivide the problem region into a few groups which act as group centers for which the interactions are computed. A matrix factorization is created from the analytic element wise expansion. This makes the representation of the Green's function in terms of matrix products possible for the iterative solver, for the case when an iterative solver can be applied to the problem 
at hand. We assume that for a coefficient evaluation the source point $\boldsymbol{r}^{\prime}$ and an observations point $r$ are well separated. Under this condition we can apply the Gegenbauer addition theorem. Also, we assume that we have chosen the order $L$ of the multipole expansion. Then, the integration on the unit sphere is performed with a numerical Gaussian quadrature with $L+1$ points below in (3.1) for the integral over $\theta$ and $2(L+1)$ points for the integral over $\phi$. We use an auxiliary variable $u=\cos \theta=\hat{\boldsymbol{k}} \cdot \hat{\boldsymbol{R}}$ and $w_{u}$ and $w_{p h i}$ are row vectors of the corresponding weights, $\Delta_{t}$ and $\Delta_{\phi}$ which are the stretching factors due to fact that the integration domain is not a unit square. The function $\alpha_{L}(k R, \hat{\boldsymbol{k}} \cdot \hat{\boldsymbol{R}})$ in (3.2) can be represented as a matrix of order $(L+1) \times(2 L+2)$ when the vector $k$ varies on the unit sphere. Further, the function $\Psi=e^{j k \cdot d}$ can be represented as a matrix of the same size. Then, the evaluation of the Green's function will be approximated as

$$
\frac{e^{j k|\boldsymbol{R}+\boldsymbol{d}|}}{|\boldsymbol{R}+\boldsymbol{d}|} \simeq \frac{j k}{4 \pi} \int_{S^{2}} e^{j k \cdot d_{\alpha_{L}}}(k R, \hat{k} \cdot \hat{\boldsymbol{B}}) d^{2} \hat{\boldsymbol{k}}
$$

The Green's functions is a key part of evaluation of the PEEC circuit parameters which are the partial inductances and the potential coefficients. The fast and accurate computation is a key aspect for the analysis of complex problems involving a large number of unknowns. Assume that the target inductive cell $m$ lies in a sphere, or group, $G_{a}$ with center $r_{a}$ and the source inductive cell $n$ lies in a sphere, or group, $G_{b}$ with its center at $r_{b}$. If the Green's function approximation is used a multipolar form for $\mathrm{dy}$ namic partial inductances and potential coefficients is obtained [3].

$$
\begin{aligned}
& L p_{m n} \simeq \frac{j k \mu \cos \theta_{m n}}{(4 \pi)^{2}} \int_{S^{2}} {\left[\frac{1}{s_{m}} \int_{v_{n}} e^{j k \cdot\left(\boldsymbol{r}_{m}-\boldsymbol{r}_{a}\right)} d v_{m}\right] } \\
& \alpha_{L, a b}\left(\hat{r}_{a b} \cdot \hat{k}\right) \\
& \\
& {\left[\frac{1}{s_{n}} \int_{v_{t h}} e^{j k \cdot\left(\boldsymbol{r}_{n}-\boldsymbol{r}_{b}\right)} d v_{n}\right] d^{2} \hat{\boldsymbol{k}}(}
\end{aligned}
$$

Here, $s_{m, n}$ are the cross sections of the volume cells for the partial inductance $m$ and $n$. If two auxiliary functions are defined as the volume integrals

$$
\begin{aligned}
& F_{m}(\boldsymbol{k})=\frac{1}{s_{m}} \int_{v_{m}} e^{j \boldsymbol{k} \cdot\left(\boldsymbol{r}_{m}-\boldsymbol{r}_{a}\right)} d v_{m} \\
& \tilde{F}_{n}(\boldsymbol{k})=\frac{1}{s_{m}} \int_{v_{n}} e^{-j \boldsymbol{k} \cdot\left(\boldsymbol{r}_{n}-\boldsymbol{r}_{b}\right)} d v_{n}
\end{aligned}
$$

Equation (3.2) can be rewritten in a more compact form as

$$
L p_{m n} \simeq \frac{j k \mu \cos \theta_{m n}}{(4 \pi)^{2}} \int_{S^{2}} F_{m}(\boldsymbol{k}) \alpha_{L, a b}\left(\hat{\boldsymbol{r}}_{a, b} \cdot \hat{\boldsymbol{k}}\right) \tilde{F_{n}}(\boldsymbol{k}) d^{2} \hat{k}
$$

It is worth to note that functions $F_{m}(\boldsymbol{k})$ and $\tilde{F}_{n}(\boldsymbol{k})$ can be evaluated just once in the beginning and re-used for each computation involving inductive cells $m$ and $n$. The volume integrals in (3.3) and (3.4) are computed by means of a Gauss-Legendre numerical integration method.

The same procedure outlined above can be applied to the computation of mutual potential coefficients $P_{m n}$. We can find

$$
\begin{aligned}
& P n_{m n} \simeq \frac{j k}{(4 \pi)^{2} \epsilon} \int_{S^{2}}\left[\frac{1}{A_{m}} \int_{A_{m}} e^{j k \cdot\left(\boldsymbol{r}_{m}-\boldsymbol{r}_{a}\right)} d A_{m}\right] \\
& \alpha_{L, a b}\left(\hat{\boldsymbol{r}}_{a b} \cdot \hat{k}\right) \\
& {\left[\frac{1}{A_{n}} \int_{A_{n}} e^{j \boldsymbol{k} \cdot\left(\boldsymbol{r}_{n}-\boldsymbol{r}_{b}\right)} d A_{n}\right] d^{2} \hat{\boldsymbol{k}} }
\end{aligned}
$$

where $A_{m, n}$ represents the surface area of the capacitive cells $m$ and $n$. It is evident again that the evaluation of the coefficient can be divided into three parts. They only share in the group data with each other but they lead to the evaluation at multiple source points $r^{\prime}$ and observation points $r$. If we define the integrals in bracket in (3.6) as $S_{m}$ and $\tilde{S}_{n}$ then the normalized coefficient of potential simplifies to

$$
P n_{m n} \simeq \frac{j k}{(4 \pi)^{2} \epsilon} \int_{S^{2}} S_{m}(k) \alpha_{L, a b}\left(\hat{\boldsymbol{r}}_{a b} \cdot \hat{k}\right) \bar{S}_{n}(\boldsymbol{k}) d^{2} \hat{k}
$$

similarly to the partial inductance (3.5).

\section{Some numerical results}

We give some results which exemplify both the FMM and FMF-based approaches for PEEC parameters computation for partial mutual inductances e.g. (3.2). Of course, more comprehensive experimentation is required to explore the entire solution space especially for general purpose EM solvers. We use a standard Gauss-Legendre (GL) integration method for both approaches. Here, we concentrate on $L_{p}$ since the volume integrals are more time consuming due to the six fold integrals. We use a GL with an adaptive choice of the order (ad-VA) where all integrals are evaluated numerically. The GL integration is compared with an analytical integration in the current direction and an adaptive GL integration in the cross-sectional dimension (ad-FA). Hence, only four for the six integrals are performed numerically. The partial mutual inductances must lead to favorable compute time results for various distances between the groups and the cells for FMM. It is clear that the quadrature aspects for the integrals are similar for both solution methods, but they are more important for the FMF approach. For FMM two inductive cells $m$ and $n$ belonging to groups $a$ and $b$ respectively. When using the FMM, by using the same notation as before $r-r^{\prime}=\boldsymbol{R}+\boldsymbol{d}$, it is assumed that $\boldsymbol{d}$ is small enough 


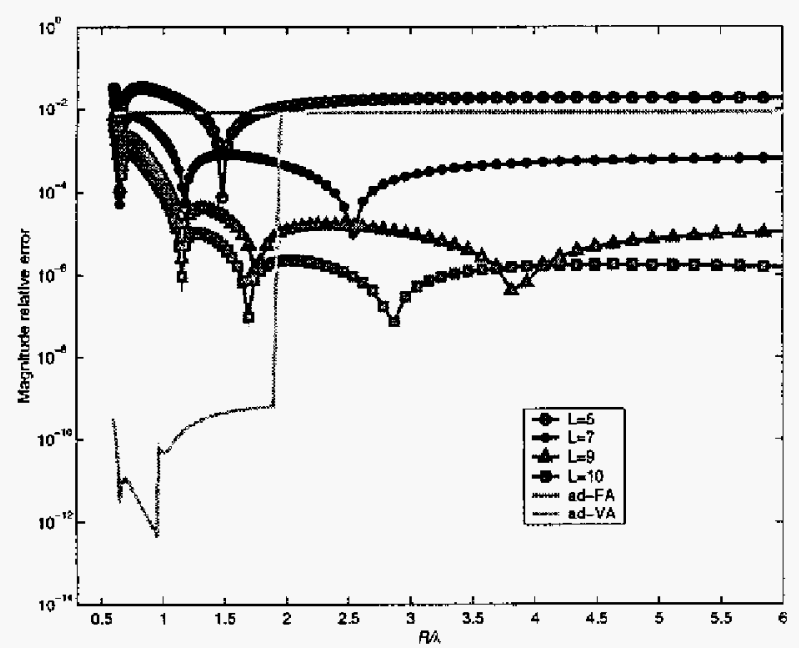

Figure 1: Comparison of relative magnitude error in $L p$ for different approaches

Table 1: Speed-up and mean relative error

\begin{tabular}{|c|c|c|}
\hline Method & speed-up & MRE \\
\hline ad-FA & 4402 & $8.7 \mathrm{e}-003$ \\
\hline ad-VA & 285 & $2.3 \mathrm{e}-003$ \\
\hline
\end{tabular}

such that $R$ is close to $r-r^{\prime}$ and where $R$ is the group center to center distance. Also, $d$ is the sum of the two local vectors to the center of the groups where the group center locations have been chosen such that $\boldsymbol{R}=R \hat{z}$ and $d=0.4 \lambda \hat{\tilde{z}}$. A maximum expansion order $L=10$ has been adopted for the FMM. The relative error magnitude in the evaluation of $L p_{m n}$ with order of expansion in the range $5-10$ assuming $R=R \hat{z}$ are shown in Fig. 1. A similar evaluation is also given for the phase error in Fig. 2.

Next, we compare the accuracy of the coefficients for the different methods. It is evident from the magnitude plot given in Fig. 1 and the phase plot in Fig. 2 that the adaptive volume integration algorithm (ad-VA) provides good accuracy even for very small electrical distances while the adaptive filament algorithm (ad-FA) provides an almost constant accuracy in magnitude which is about equivalent to a 7 -th order FMM for the phase error. The ad-FA approach is an example of a mixed analytic function and numerical solution approach. The ad-FA solution is faster than the ad-VA approach as a consequence of the reduction in dimensionality of the numerical integration solution. Table 1 shows the speed-up achieved and the mean relative error (MRE) for the ad-FA and the ad-VA as compared to a 9 -th order Gauss-Legendre integration (GL-9) result. Of course the efficiency of all these approaches are very much dependent on the implementation details. In

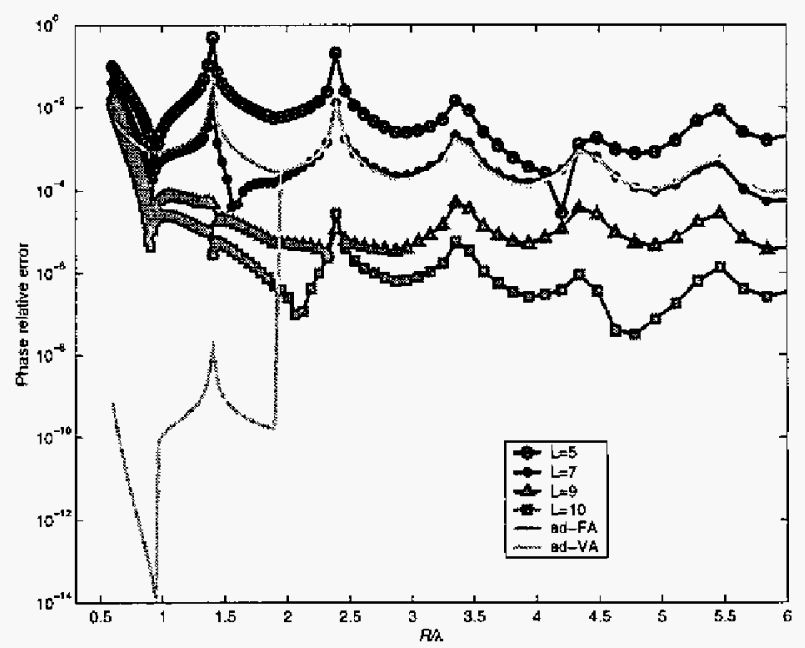

Figure 2: Comparison of relative phase error in $L p$ for different approaches

Table 1 we show that, for a favorable case, the ad-FA approach can lead to a good speed-up with good accuracy over straight forward numerical volume integration. We found in general that the integration order $L$ does not significantly impact the compute time while it is very important for highly accurate coefficients.

\section{Conclusion}

In this short paper we did outline methods for speeding up the fast circuit element evaluation for the fast multi pole FMM and the fast multi-function FMF methods. However, for general purpose combined circuit and $\mathrm{EM}$ solvers, the ranges of applicability still needs to be explored to come up with the best methods.

\section{References}

[1] V. Rokhlin. Rapid Solution of Integral Equations of Scattering Theory in Two Dimensions. Journal of Computational Physics, 86(2):414-439, 1990.

[2] J. M. Song, W. C. Chew. Multilevel Fast-Multipole Algorithm for Solving Combined Field Integral Equations of Electromagnetic Scattering. Microwave and Optical Technology Letters, 10(1):14-19, September 1995.

[3] G. Antonini. The Fast Multipole Method for PEEC Circuits Analysis. In Proc of the IEEE Int. Symp. on Electromagnetic Compatibility, pages 446-451. Minneapolis, MN, August 2002. 